\title{
Effects of simvastatin on carotenoid status in plasma
}

Mireille Rydén, Per Leanderson, K-O Kastbom and Lena Jonasson

\section{Linköping University Post Print}

N.B.: When citing this work, cite the original article.

Original Publication:

Mireille Rydén, Per Leanderson, K-O Kastbom and Lena Jonasson, Effects of simvastatin on carotenoid status in plasma, 2012, NMCD. Nutrition Metabolism and Cardiovascular Diseases, (22), 1, 66-71.

http://dx.doi.org/10.1016/j.numecd.2010.04.009

Copyright: Elsevier

http://www.elsevier.com/

Postprint available at: Linköping University Electronic Press

http://urn.kb.se/resolve?urn=urn:nbn:se:liu:diva-59339 
Effects of simvastatin on carotenoid status in plasma.

Rydén $\mathrm{M}^{\mathrm{a}}$, Leanderson $\mathrm{P}^{\mathrm{b}}$, Kastbom K-O $\mathrm{O}^{\mathrm{a}}$, Jonasson $\mathrm{L}^{\mathrm{a}}$

${ }^{\mathrm{a}}$ Division of Cardiovascular Medicine, Department of Medical and Health Sciences, Faculty of Health Sciences, Linköping University, SE-581 85 Linköping, Sweden.

${ }^{\mathrm{b}}$ Division of Occupational and Environmental Medicine, Department of Molecular and Clinical Medicine, Faculty of Health Sciences, Linköping University, SE-581 85 Linköping, Sweden.

Correspondence: Lena Jonasson, $\mathrm{MD}, \mathrm{PhD}$, Division of Cardiovascular Medicine, Department of Medical and Health Sciences, Linköping University, SE-581 85 Linköping, Sweden. Tel: +46 132251 94, fax +46131491 06, e-mail: lena.jonasson@lio.se

Word counts: Abstract: 229, Text: 1992, Number of references: 30, Number of tables: 4, Number of figures: 1 . 


\section{Key words}

Statin; carotenoid; lipid; lipoprotein; inflammation.

\section{Acronyms}

$\begin{array}{ll}\text { ApoB } & \begin{array}{l}\text { apolipoprotein B } \\ \text { BMI }\end{array} \\ \text { CRP } & \text { Body Mass Index } \\ \text { HDL } & \text { high density lipoprotein } \\ \text { IL-6 } & \text { interleukin-6 } \\ \text { LDL } & \text { low density lipoprotein } \\ \text { ox-LDL } & \text { oxidized LDL }\end{array}$




\begin{abstract}
Background and Aims. Carotenoids are potent antioxidants mainly transported in the low density lipoprotein (LDL) fraction. They may also influence the immune response and inverse associations with inflammatory markers have been reported. We investigated whether simvastatin, by exerting both lipid-lowering and anti-inflammatory effects, altered the carotenoid status in plasma.
\end{abstract}

Methods and Results. A randomized, double-blind, placebo-controlled study design was applied. Eighty volunteers with mild to moderate hypercholesterolemia received either simvastatin $40 \mathrm{mg}$ or placebo for 6 weeks. Lipids, oxidized LDL (ox-LDL), C-reactive protein (CRP), interleukin (IL)-6, oxygenated carotenoids (lutein, zeaxanthin, $\beta$ cryptoxanthin) and hydrocarbon carotenoids ( $\alpha$-carotene, $\beta$-carotene, lycopene) were measured in plasma. Simvastatin use was associated with significant reductions in total cholesterol, LDL, ox-LDL and CRP. Simvastatin therapy also resulted in reduced plasma levels of both oxygenated and hydrocarbon carotenoids. However, when adjusted for lipids, all carotenoids except $\beta$-cryptoxanthin showed significant increases after simvastatin therapy. Both crude and lipid-adjusted carotenoids were inversely correlated with CRP and IL-6 in plasma but the change in carotenoid status during simvastatin therapy was not specifically related to any changes in inflammatory markers.

Conclusions. To summarize, the change in carotenoid status during simvastatin therapy was mainly attributed to the lowering of cholesterol and not to the suppression of inflammatory activity. After adjustment for lipids, the levels of lutein, lycopene, $\alpha$-carotene and $\beta$-carotene 
were significantly increased by simvastatin suggesting an increased ratio of carotenoids per particle. 


\section{Introduction}

The oxidative modification of lipoproteins in the arterial wall is considered to play an important role in the inflammatory process of atherosclerosis and it is well established that circulating levels of ox-LDL are independently predictive of both subclinical atherosclerosis and myocardial infarction $(1,2)$. The resistance of LDL to oxidation is influenced by several factors, among which the content of fat-soluble vitamins and carotenoids is prominent. In particular, carotenoids have shown to be of importance. In a recent study comparing $\alpha$ tocopherol, retinol and carotenoids, plasma carotenoids were the most powerful determinants of in vivo oxidatively modified LDL (3). Moreover, during LDL oxidation in vitro, $\alpha$ - and $\beta$ carotene are consumed at a significantly higher rate than $\alpha$ - or $\gamma$-tocopherol (4).

Carotenoids are a group of lipophilic phytochemicals that exist in nature in large variety. The major ones detectable in human plasma are $\alpha$-carotene, $\beta$-carotene and lycopene (hydrocarbon carotenoids) and lutein, zeaxanthin and $\beta$-cryptoxanthin (oxygenated carotenoids) (5). Inverse associations between plasma levels of carotenoids and cardiovascular disease have been reported in several studies (6-10). The majority of studies has focused on $\beta$-carotene and lycopene while a few reports have suggested that oxygenated carotenoids may be of even greater importance for atheroprotection (11-13). Interestingly, carotenoids also exert positive influences on the immune response which may be, at least in part, mediated by their antioxidant effects (14). Both in population-based studies and in studies of coronary patients, inverse associations between circulating carotenoids and markers of systemic inflammation have been reported (15-21).

Hydroxymethylglutaryl-coenzyme A reductase inhibitors (statins) are cholesterol-lowering drugs that markedly reduce cardiovascular mortality and morbidity (22). Their benefit may 
not only be attributed to lipid-lowering but also to non-lipid dependent, so-called pleiotropic effects. During recent years, considerable attention has been drawn to a number of antiinflammatory and immunomodulatory effects shown in vitro and in animal models $(23,24)$. The clinical relevance of these effects are not fully proven but it has been consistently shown that statin treatment leads to significant reductions in circulating C-reactive protein (CRP), thus supporting an anti-inflammatory effect in vivo $(25,26)$.

Given both the lipid-lowering and anti-inflammatory effects exerted by statins, it may be hypothesized that these agents also influence the levels of carotenoids in plasma. The aim of this study was to clarify whether simvastatin treatment altered the carotenoid status in plasma, including oxygenated and hydrocarbon carotenoids.

\section{Methods}

\section{Participants and study design}

As has been described earlier (27), the study population consisted of 80 middle-aged men with mild to moderate hypercholesterolemia. They were clinically healthy receiving no medication. A randomized, double blind and placebo-controlled study design was applied after approval by the ethical committee. Individuals gave informed consent and were randomly assigned to receive either $40 \mathrm{mg}$ simvastatin daily or matching placebo tablets. They were instructed to maintain their habitual dietary pattern during the study period. Compliance with study treatment was assessed at each follow-up by reviewing the calendarpacked tablets remaining. Fasting venous blood was collected at baseline and after 6 weeks.

Lipid profile, apolipoprotein B, ox-LDL and inflammatory markers 
Plasma levels of cholesterol, LDL cholesterol, high density lipoprotein (HDL) cholesterol, triglycerides and apolipoprotein (apo)B were determined as earlier outlined (27). Ox-LDL in plasma was measured using a competitive ELISA (Mercodia Oxidized LDL, Uppsala, Sweden) with a lower detection limit of $<1$ unit/l. The inter-assay coefficient of variation for ox-LDL was $<10 \%$. CRP was determined by using a highly sensitive latex-enhanced turbidimetric immunoassay with a lower detection limit of $0.03 \mathrm{mg} / \mathrm{L}$ (Roche Diagnostics GmbH, Vienna, Austria). IL-6 was measured by an ELISA immunoassay (Quantikine HS, R \& D Systems Europe Ltd, Abingdon, United Kingdom) with a lower detection limit of 0.04 $\mathrm{pg} / \mathrm{mL}$. The inter-assay coefficients of variation for CRP and IL-6 were $<5 \%$.

\section{Carotenoids}

Plasma levels of carotenoids (lutein, zeaxanthin, $\beta$-cryptoxanthin, lycopene, $\alpha$-carotene, $\beta$ carotene) were determined with high-performance liquid chromatography as previously described (19). Since lutein is almost structurally identical to its stereo isomer zeaxanthin, the analysis does not discriminate between them and the sum of these carotenoids is presented as a single value (zeaxanthin normally representing less than $15 \%$ of this value). The intra- and interassay coefficient of variation varied between 5 and $7 \%$. The validity of the method was ensured by including a reference sample that was calibrated against an external standard (SRM 968C) from the National Institute of Standard and Technology (19). The plasma levels of carotenoids were expressed as non-adjusted (crude) or adjusted for total cholesterol, LDL cholesterol or apoB levels.

Statistical analyses: The SPSS 17.0 was used for statistical analyses. Differences within the groups were analyzed using Wilcoxon signed-rank or Chi-Square tests. A two-way repeated ANOVA was used for comparisons between groups. We used the Spearman rank correlation 
test to evaluate the relationships between the variables. Two-tailed p-value $<0.05$ were considered significant.

\section{Results}

Complete data were obtained from 79 of the 80 participants. Three individuals suffered from inflammatory or infectious disorders during the study period but otherwise, no important adverse effects or side effects occurred. In all, data were obtained and analysed from 39 individuals in the placebo group and 37 in the simvastatin group. The study medication was well tolerated and the compliance was as high in the simvastatin group as in the placebo group ( $89 \%$ and $91 \%$, respectively). The baseline characteristics are outlined in Table 1 . The lipid and inflammatory variables before and after 6 weeks are presented in Table 2. The changes in total cholesterol, LDL cholesterol, apoB, ox-LDL and CRP within the simvastatin group remained significant when they were compared with the placebo group.

After 6 weeks the crude plasma levels of oxygenated (lutein+zeaxanthin and $\beta$-cryptoxanthin) and hydrocarbon carotenoids (lycopene, $\alpha$-carotene and $\beta$-carotene) were reduced in the simvastatin group by $21 \%(\mathrm{p}<0.001)$ and $5.2 \%(\mathrm{p}=0.05)$, respectively. No significant reductions of oxygenated or hydrocarbon carotenoids were seen in the placebo group. On the other hand, when the levels of carotenoids were adjusted for total cholesterol, the treatment with simvastatin resulted in increased levels of all carotenoids (except $\beta$ cryptoxanthin) that were significant both within the group and between groups (Table 3). Similar results were obtained in the simvastatin group when individual carotenoids were adjusted to LDL cholesterol (data not shown) or apoB. The changes in apoB-adjusted levels of oxygenated and hydrocarbon carotenoids are illustrated in Figure 1. 
Crude or lipid-adjusted plasma levels of carotenoids did not correlate with any clinical characteristics, such as body mass index (BMI) or smoking status. There was a significant correlation between crude levels of oxygenated carotenoids and total cholesterol that remained after 6 weeks $(r=0.56, \mathrm{p}<0.001)$. The correlations between crude levels of oxygenated carotenoids and LDL cholesterol also remained significant after 6 weeks $(r=$ $0.49, \mathrm{p}<0.001)$. Similarly, the significant correlations between hydrocarbon carotenoids and total cholesterol or LDL cholesterol remained unchanged during simvastatin therapy $(r=$ $0.33, \mathrm{p}<0.01$ and $r=0.29$ respectively, $\mathrm{p}<0.05)$. At baseline, none of the carotenoids correlated with HDL cholesterol, triglycerides or apoB. After 6 weeks of simvastatin treatment, both oxygenated and hydrocarbon carotenoids correlated with HDL cholesterol $(r=$ $0.42, \mathrm{p}<0.001$ and $r=0.38, \mathrm{p}<0.01$, respectively) whereas only oxygenated carotenoids correlated with apoB $(r=0.38, \mathrm{p}<0.01)$. The relative change in oxygenated carotenoids was significantly associated with the relative changes in total cholesterol and apoB (both $\mathrm{p}<0.01$ ) but otherwise, there were no correlations between the longitudinal changes of lipids and carotenoids. Carotenoids and ox-LDL were not correlated at any time point.

The levels of CRP and IL-6 correlated both at baseline and after 6 weeks $(r=0.40, \mathrm{p}<0.01)$. The inflammatory markers were not correlated with any of the lipid variables, including oxLDL. As shown in Table 4, the baseline levels of crude and lipid-adjusted levels of all single carotenoids showed inverse correlations with CRP and IL-6. The associations between carotenoids and inflammatory markers were however attenuated during simvastatin therapy except for $\beta$-carotene that remained inversely correlated with CRP (crude, $r=-0.35$, lipidadjusted, $r=-0.37$, both $\mathrm{p}<0.05$, respectively). 


\section{Discussion}

In the present study, the crude plasma levels of carotenoids fell significantly in parallel with LDL and total cholesterol during simvastatin therapy. However, when adjusted for total cholesterol, LDL cholesterol or apoB levels, all individual carotenoids (except $\beta$ cryptoxanthin) showed significant increases in the simvastatin group. The findings may indicate that atherogenic lipoprotein particles have improved their antioxidant status through enrichment of carotenoids during simvastatin therapy. Vasankari et al (28) showed that $\beta$ carotene in serum decreased during the first 12 weeks of simvastatin therapy $(20 \mathrm{mg} / \mathrm{d})$ while the $\beta$-carotene:LDL cholesterol ratio increased after 12 and 52 weeks of therapy in parallel with an increased antioxidant capacity of LDL particles. Shin et al (29) also reported decreased plasma levels of $\alpha$ - and $\beta$-carotene after 8 weeks of simvastatin therapy (20-40 $\mathrm{mg} / \mathrm{d}$ ) but, in contrast to our present findings and the study by Vasankari et al (28), they were not able to show any changes in $\alpha$-and $\beta$-carotene when levels were adjusted for lipids. Circulating levels of carotenoids, without adjustment for lipids, are well-established markers of cardiovascular risk (6-13) but apparently, there is a risk to misinterpret the carotenoid status in individuals with cholesterol-lowering therapy if relying on crude serum or plasma levels.

The plasma concentrations of major carotenoids showed inverse and significant associations with CRP and IL-6 levels. The findings are in line with previous population-based studies reporting inverse associations between carotenoids in plasma and CRP after adjustment for confounding factors like dietary intake and smoking (15-21). Various explanations have been given, such as the consumption of circulating carotenoids during the neutralization of free radicals or depletion from blood due to an increased uptake by immune cells. It has also been 
emphasized that crude levels of plasma carotenoids may give misleading information in the presence of a systemic inflammatory response. In a previous study by Gray et al (30), the time course of the change in plasma carotenoids was studied in 30 patients following elective surgery. During the first days, CRP levels increased while plasma concentrations of several major carotenoids, including $\beta$-carotene and lutein, fell significantly. However, when carotenoids were normalized to cholesterol levels, the reductions were no longer statistically significant. In the present study, we can clearly show that not only crude plasma levels but also lipid-adjusted levels of individual major carotenoids are inversely correlated with both CRP and IL-6 in a clinically healthy population.

There is more than one reason to believe that statins have an influence on circulating levels of carotenoids. Besides their potent lipid-lowering effects, pleiotropic effects in vivo include significant reductions in CRP which appear to be independent of the lipid-lowering capacity $(25,26)$. However, in the present study, the change in carotenoid status during simvastatin therapy was not specifically related to any changes in inflammatory activity. Among individual carotenoids, only $\beta$-carotene still showed a weak but significant association with CRP during treatment. The findings are in agreement with several large population-based studies showing that, among carotenoids, $\beta$-carotene have the strongest relationship with inflammatory markers $(15,16,18,21)$. In a previous study, we measured a panel of lipophilic antioxidants in patients with coronary artery disease, many of whom were receiving long-term statin treatment, and found that inflammatory activity was inversely associated with serum $\beta$ carotene but not with lycopene or other antioxidants (17). 
One limitation of our study is the rather small sample size. Another limitation concerns the duration of treatment. The effects of long-term simvastatin treatment remain unknown. Third, an assay for measuring the resistance of LDL to oxidation would have been of value.

To summarize, the change in carotenoid status during simvastatin therapy was mainly attributed to the lowering of cholesterol and not to the suppression of inflammatory activity. The crude plasma levels of both oxygenated and hydrocarbon carotenoids were reduced by simvastatin. However, when adjusted for lipids, the levels of lutein, lycopene, $\alpha$-carotene and $\beta$-carotene were significantly increased by simvastatin suggesting an increased ratio of carotenoids per particle.

\section{Disclosure statement}

None of the authors have any actual or potential conflict of interest to disclose.

\section{Role of the Funding Sources}

This work was funded by grants from the Swedish Heart-Lung Foundation. Study medication was provided by Merck Sharp \& Dohme. None of the study sponsors had any role in the design, conduct of the trial or analysis of the data. 


\section{References}

1. Hulthe J, Fagerberg B. Circulating oxidized LDL is associated with subclinical atherosclerosis development and inflammatory cytokines (AIR Study). Arterioscler Thromb Vasc Biol 2002;22:1162-1167.

2. Meisinger C, Baumert J, Khuseyinova N, Loewel H, Koenig W. Plasma oxidized lowdensity lipoprotein, a strong predictor for acute coronary heart disease events in apparently healthy, middle-aged men from the general population. Circulation 2005;112:651-657.

3. Karppi J, Nurmi T, Kurl S, Rissanen TH, Nyyssönen K. Lycopene, lutein and $\beta$ carotene as determinants of LDL conjugated dienes in serum. Atherosclerosis 2009; epub ahead of print.

4. Kontush A, Weber W, Beisiegel U. $\alpha$ - and $\beta$-carotenes in low density lipoproteins are the preferred target for nitric oxide-induced oxidation. Atherosclerosis 2000;148:8793.

5. Britton G. Structure and properties of carotenoids in relation to function. FASEB J $1995 ; 9: 1551-1558$.

6. Rissanen TH, Voutilainen S, Nyyssönen K, Lakka T, Sivenius J, Salonen R, et al. Low serum lycopene concentrations is associated with an excess incidence of acute coronary events and stroke: the Kuopi Ischemic Heart Disease Risk Factor Study. Br J Nutr 2001;85:749-754.

7. Buijsse B, Feskens EJ, Kwape L, Kok FJ, Kromhout D. Both alpha- and beta-carotene, but not tocopherols and vitamin $\mathrm{C}$, are inversely related to 15 -year cardiovascular mortality in Dutch elderly men. J Nutr 2008;138:344-350. 
8. Ito Y, Kurata M, Suzuki K, Hamajima N, Hishida H, Aoki K. Cardiovascular disease mortality and serum carotenoid levels: a Japanese population-based follow-up study. J Epidemiol 2006;16:154-160.

9. Riccioni G, D’Orazio N, Palumbo N, Bucciarelli V, Ilio E, Bazzano LA et al. Relationship between plasma antioxidant concentrations and carotid intima-media thickness: the Asymptomatic Carotid Atherosclerotic Disease in Manfredonia Study. Eur J Cardiovasc Prev Rehabil 2009;16:351-357.

10. Hosawa A, Jacobs DR, Steffes MW, Gross MD, Steffen LM, Lee D-H. Circulating carotenoid concentrations and incident hypertension: the Coronary Artery Risk Development in Young Adults (CARDIA) study. J Hypertens 2009;27:237-242.

11. Dwyer JH, Navab M, Dwyer KM, Hassan K, Sun P, Shircore A, et al. Oxygenated carotenoid lutein and progression of early atherosclerosis: the Los Angeles atherosclerosis study. Circulation 2001;103:2922-2927.

12. Dwyer JH, Paul-Labrador MJ, Fan J, Shircore AM, Merz CN, Dwyer KM. Progression of carotid intima-media thickness and plasma antioxidants: the Los Angeles Atherosclerosis Study. Arterioscler Thromb Vasc Biol 2004;24:313-319.

13. Koh W-P, Yuan J-M, Wang R, Lee Y-P, Lee B-L, Yu MC, Ong C-N. Plasma carotenoids and risk of acute myocardial infarction in the Singapore Chinese Health Study. Nutr Metab Cardiovasc Dis 2010; Epub ahead of print.

14. Chew BP, Park JS. Carotenoid action on the immune response. J Nutr 2004;134:257S$261 \mathrm{~S}$.

15. Kritchevsky SB, Bush AJ, Pahor M, Gross MD. Serum carotenoids and markers of inflammation in nonsmokers. Am J Epidemiol 2000;152:1065-1071. 
16. Erlinger TP, Guallar E, Miller ER $3^{\text {rd }}$, Stolzenberg-Solomon R, Appel LJ. Relationship between systemic markers of inflammation and serum beta-carotene levels. Arch Intern Med 2001;161:1903-1908.

17. Jonasson L, Wikby A, Olsson AG. Low serum beta-carotene reflects immune activation in patients with coronary artery disease. Nutr Metab Cardiovasc Dis 2003; 13:120-125

18. van Herpen-Broekmans WM, Klöpping-Ketelaars IA, Bots ML, Kluft C, Princen H, Hendriks HF, Tijburg LB, et al. Serum carotenoids and vitamins in relation to markers of endothelial function and inflammation. Eur J Epidemiol 2004;19:915-921.

19. Lidebjer C, Leanderson P, Ernerudh J, Jonasson L. Low plasma levels of oxygenated carotenoids in patients with coronary artery disease. Nutr Metab Cardiovasc Dis 2007;17:448-456.

20. Hosawa A, Jacobs DR, Steffes MW, Gross MD, Steffen LM, Lee D-H. Relationships of circulating carotenoid concentrations with several markers of inflammation, oxidative stress, and endothelial dysfunction: The Coronanry Artery Risk Development in Young Adults (CARDIA)/Young Adult Longitudinal Trends in Antioxidants (YALTA) Study. Clin Chem 2007;53:447-455.

21. Wang L, Gaziano JM, Norkus EP, Buring JE, Sesso HD. Associations of plasma carotenoids with risk factors and biomarkers related to cardiovascular disease in middle-aged and older women. Am J Clin Nutr 2008;88:747-754.

22. Baigent C, Keech A, Kearney PM, Blackwell L, Buck G, Pollicino C, et al; Cholesterol Treatment Trialists'(CTT) Collaborators. Efficacy and safety of cholesterol-lowering treatment: prospective meta-analysis of data from 90,056 participants in 14 randomized trials of statins. Lancet 2005;366:1267-1278. 
23. Kwak BR, Mulhaupt F, Mach F. Atherosclerosis: anti-inflammatory and immunomodulatory activities of statins. Autoimmunity Rev 2003;2:332-338

24. Forrester JS, Libby P. The inflammation hypothesis and its potential relevance to statin therapy. Am J Cardiol 2007;99:732-738.

25. Jialal I, Stein D, Balis D, Grundy SM, Adams-Huet B, Devaraj S. Effect of hydroxymethyl glutaryl coenzym A reductase inhibitor therapy on high sensitive Creactive protein levels. Circulation 2001;103:1933-1935

26. Ridker PM, Danielson E, Fonseca FAH, Genest J, Gotto AM, Kastelein JJP, et al, for the JUPITER Study group. Rosuvastatin to prevent vascular events in men and women with elevated C-reactive protein. N Engl J Med 2008;359:2195-2207.

27. Cherfan P, Tompa A, Wikby A, Löfgren S, Jonasson L. Effects of simvastatin on human T cells in vitro. Atherosclerosis 2007;193:186-192.

28. Vasankari T, Ahotupa M, Viikari J, Nuotio I, Strandberg T, Vanhanen H, et al. Effect of 12-month statin therapy on antioxidant potential of LDL and serum antioxidant vitamin concentrations. Ann Med 2004; 36:618-622.

29. Shin MJ, Chung N, Lee JH, Jang Y, Park E, Jeon KI, et al. Effects of simvastatin on plasma antioxidant status and vitamins in hypercholesterolemic patients. Int Journal Cardiol 2007:118;173-177.

30. Gray A, McMillan DC, Wilson C, Williamson C, O’Reilly DSJ, Talwar D. The relationship between the acute changes in the systemic inflammatory response, lipid soluble antioxidant vitamins and lipid peroxidation following elective knee arthroplasty. Clin Nutr 2005;24:746-750. 